\title{
IncRNA TINCR attenuates the proliferation and invasion, and enhances the apoptosis of cutaneous malignant melanoma cells by regulating the miR-424-5p/LATS1 axis
}

\author{
XUEMEI HAN $^{1 *}$, YUXI JIA $^{2 *}$, XIANGRU CHEN ${ }^{2}, \mathrm{CHENGKUAN} \mathrm{SUN}^{3}$ and JING SUN ${ }^{2}$ \\ Departments of ${ }^{1}$ Neurology, ${ }^{2}$ Dermatology and ${ }^{3}$ Handsurgery, The China-Japan Union Hospital of Ji Lin University, \\ Changchun, Jilin 130033, P.R. China
}

Received April 9, 2021; Accepted August 10, 2021

DOI: $10.3892 /$ or.2021.8189

\begin{abstract}
Cutaneous malignant melanoma (CMM) is responsible for $\geq 1 / 2$ of skin cancer-related mortalities. The aberrant expression of long non-coding RNAs (lncRNAs) has been associated with the development of CMM. However, to the best of our knowledge, the role of the IncRNA TINCR ubiquitin domain containing (TINCR) in CMM has not been previously investigated, and thus, the current study aimed to evaluate this in vitro and in vivo. Reverse transcription-quantitative $\mathrm{PCR}$ (RT-qPCR) was used to analyze microRNA (miR)-424-5p expression, and RT-qPCR and western blotting were used to measure TINCR, large tumor suppressor kinase 1 (LATS1), cellular communication network factor 2 (CTGF), cellular communication network factor 1 (CCN1) and AXL receptor tyrosine kinase (AXL) mRNA and protein expression levels, respectively. Cell Counting Kit-8, flow cytometry and Transwell assays were used to detect the proliferation, apoptosis and invasion of CMM cell lines, respectively. The binding sites between TINCR and miR-424-5p were predicted using the miRDB database. A dual luciferase reporter assay and RT-qPCR were used to identify the relationship between TINCR and miR-424-5p in CMM cell lines. The bioinformatics analysis revealed that TINCR was one of the most significantly downregulated lncRNAs in CMM, and advanced stage CMM tissues showed the greatest decrease in TINCR expression. Moreover, in the collected CMM tissues and tested cell lines of the current study, TINCR expression was found to be downregulated compared with the respective controls.
\end{abstract}

Correspondence to: Dr Jing Sun, Department of Dermatology, The China-Japan Union Hospital of Ji Lin University, 126 Xiantai Street, Changchun, Jilin 130033, P.R. China

E-mail: sunjing99@jlu.edu.cn

*Contributed equally

Key words: cutaneous malignant melanoma, long non-coding RNA TINCR ubiquitin domain containing, microRNA-424-5p/large tumor suppressor kinase 1 axis, cell proliferation, cell apoptosis
Notably, TINCR overexpression inhibited the expression levels of CTGF, CCN1 and AXL, decreased the proliferation and invasion, and induced the apoptosis of CMM cell lines. In addition, a mutual binding association was identified between miR-424-5p and TINCR in CMM cells. LATS1, a target of miR-424-5p, was found to be positively regulated by TINCR. TINCR activated Hippo signaling and repressed the activity of Yes 1 associated transcriptional regulator by regulating LATS1 expression, while LATS1 knockdown reversed the effect of TINCR overexpression on CMM cells. Collectively, the findings of the present study suggested that TINCR may attenuate the progression of CMM by regulating the miR-424-5p/LATS1 signaling axis. These results indicated that TINCR may play a tumor suppressive role in CMM.

\section{Introduction}

Melanoma, which remains the leading cause of skin cancer-related mortality in patients, is characterized by high levels of metastasis (1). In 2017, the age-standardized prevalence rate of melanoma was 0.9 per 100,000 in China (2). As a type of malignant tumor, cutaneous malignant melanoma (CMM) is derived from melanocytes in the skin (3). Worldwide, the morbidity of CMM is increasing and the age of onset is decreasing (4), which may be associated with genetic factors and increased exposure to UV, amongst other risk factors (5). The majority of patients with CMM can be cured by early detection and treatment (6), including surgical resection (7) and chemo- and radiotherapy (8); however, the prognosis of patients with CMM remains poor following late detection.

Non-coding regulatory RNAs can be divided into two groups: Small non-coding RNAs ( $\leq 200$ nucleotides in length) and long non-coding RNAs (lncRNAs; >200 nucleotides in length) (9). IncRNAs regulate gene expression via numerous molecular mechanisms, including RNA degradation, microRNA (miRNA/miR) sequestration and transcriptional and translational activation or repression (10). Accumulating evidence has indicated that numerous lncRNAs play roles in the tumorigenesis of various cancer types, including CMM. For example, IncRNA forkhead box D3 antisense RNA 1 was previously discovered to increase CMM cell proliferation, invasion and migration by regulating the miR-325/mitogen-activated 
protein kinase kinase kinase 2 axis (11). In addition, the lncRNA FOXF1 adjacent non-coding developmental regulatory RNA was reported to inhibit the migration and invasion of CMM cells (12). The IncRNA TINCR ubiquitin domain containing (TINCR) was also found to function as a tumor suppressor in various types of cancer. For instance, a previous study revealed that $\mathrm{Sp1}$ transcription factor-induced upregulation of TINCR expression sponged miR-7-5p expression and promoted the development of colorectal cancer (13). It has also been shown that the activation of TINCR by the methylation of histone 3 on lysine 27 induced epithelial-mesenchymal transition in breast cancer by targeting miR-125b (14). Furthermore, TINCR was reported to serve a tumor suppressive role over the proliferation, invasion and migration of prostate cancer cells by regulating thyroid hormone receptor interactor 13 (15). However, to the best of our knowledge, the precise function of TINCR in CMM remains unknown, which will be investigated in the present study.

\section{Materials and methods}

Patient samples. In total, 60 patients with CMM (age range, 51-75 years; 46 men and 14 women) who underwent surgical resection at The China-Japan Union Hospital of Ji Lin University (Jilin, China) between December 2016 and January 2018 were enrolled in the current study. The site of CMM was distributed on the back (38 cases), head (11 cases), leg ( 7 cases), chest ( 2 cases), buttock (1 case) and hand (1 case). $\mathrm{CMM}$ and adjacent normal tissues ( $2 \mathrm{~cm}$ from tumor tissues) were extracted and immediately stored at $-80^{\circ} \mathrm{C}$. All patients provided written informed consent prior to participation in the study. The inclusion criteria were histopathological confirmation of CMM. Exclusion criteria included uncontrolled infections, autoimmune disease, the usage of systemic corticosteroids and systemic therapy for CMM before the enrollment. The current study protocol was approved by the Ethical Committee of The China-Japan Union Hospital of Ji Lin University (approval no. CJUHJLU20161102).

Cell lines and culture. Human CMM cell lines (M14, A375 and MV3) were purchased from the American Type Culture Collection. Human immortalized keratinocytes, HaCaT, were obtained from The Cell Bank of Type Culture Collection of The Chinese Academy of Sciences. HaCaT cells were authenticated by using STR profiling. Cells were cultured in RPMI-1640 medium (Gibco; Thermo Fisher Scientific, Inc.) supplemented with 10\% FBS (Gibco; Thermo Fisher Scientific, Inc.) and 1\% penicillin/streptomycin (Sigma-Aldrich; Merck KGaA), and maintained in an incubator with $5 \% \mathrm{CO}_{2}$ at $37^{\circ} \mathrm{C}$.

Bioinformatics analysis. The expression levels of TINCR in benign nevus, atypical nevus, melanoma in situ, vertical growth phase melanoma and metastatic growth phase melanoma tissues were analyzed using data obtained from the GSE4587 dataset (16) in the Gene Expression Omnibus (GEO) database (https://www.ncbi.nlm.nih.gov/gds/?term=). The expression level of TINCR in 95 individuals, representing 27 different tissues, was retrieved from the Human Protein Atlas RNA-sequencing (seq) normal tissues project published by Fagerberg et al (17). Expression data of TINCR in 558 normal and 461 skin cutaneous melanoma (SKCM) tissues deposit in The Cancer Genome Atlas (TCGA) (TCGA-SKCM) were downloaded from the Gene Expression Profiling Interactive Analysis database (http://gepia.cancer.pku.cn) (18).

Cell transfection. pcDNA3.1 empty vector (control; $2 \mu \mathrm{g}$ ), pcDNA3.1-TINCR vector $(2 \mu \mathrm{g})$, miR-424-5p mimic $(50 \mathrm{nM}$, 5'-CAGCAGCAAUUCAUGUUUUGAA-3'), miR-negative control (NC) mimic (50 nM, 5'-UCGCUUGGUGCAGGU CGGGAATT-3'), miR-424-5p inhibitor (50 nM, 5'-UCC AAAACAUGAAUUGCUGCUG-3') or miR-NC inhibitor (50 nM, 5'-UUCUCCGAACGUGUCACGUTT-3') (all from Guangzhou RiboBio Co., Ltd.) were mixed with Lipofectamine ${ }^{\circledR} 3000$ reagent (Invitrogen; Thermo Fisher Scientific, Inc.) in serum-free RPMI-1640 medium and incubated for $15 \mathrm{~min}$ at room temperature. Following the incubation, the mixture was added into each well of a 6-well plate $\left(1 \times 10^{6}\right.$ cells/well $)$ and incubated for $48 \mathrm{~h}$ at $37^{\circ} \mathrm{C}$. A total of $48 \mathrm{~h}$ after the transfection, the cells were harvested for use in subsequent experiments.

For LATS1 knockdown, small interfering RNA (si) control (siControl, $50 \mathrm{nM}, 5$ '-UUCUCCGAACGUGUCACGUTT-3') and siLATS1 (50 nM, 5'-GGUGAAGUCUGUCUAGCAA-3') (both from Guangzhou RiboBio Co., Ltd.) were mixed with Lipofectamine ${ }^{\circledast}$ RNAiMax transfection reagent (Invitrogen; Thermo Fisher Scientific, Inc.) in serum-free RPMI-1640 medium and incubated for $5 \mathrm{~min}$ at room temperature. Following the incubation, the mixture was added into each well of a 6 -well plate $\left(1 \times 10^{6}\right.$ cells/well) and incubated for $48 \mathrm{~h}$ at $37^{\circ} \mathrm{C}$. A total of $48 \mathrm{~h}$ after the transfection, the cells were harvested for use in subsequent experiments.

Reverse transcription-quantitative PCR (RT-qPCR). Total RNA was extracted from CMM tissues and cell lines using TRIzol $^{\circledR}$ (Invitrogen; Thermo Fisher Scientific, Inc.) and reverse transcribed into cDNA using a PrimeScript RT reagent kit (Takara Bio, Inc.) according to the manufacturer's instructions. qPCR was subsequently performed using SYBR Premix Ex Taq (Takara Bio, Inc.) on a CFX96 Real-Time PCR system (Bio-Rad Laboratories, Inc.). The following thermocycling conditions were used for the qPCR: Initial denaturation at $95^{\circ} \mathrm{C}$ for $1 \mathrm{~min}$; followed by 45 cycles of denaturation at $95^{\circ} \mathrm{C}$ for $15 \mathrm{sec}$, annealing at $60^{\circ} \mathrm{C}$ for $31 \mathrm{sec}$ and elongation at $72^{\circ} \mathrm{C}$ for $30 \mathrm{sec}$, and final extension at $72^{\circ} \mathrm{C}$ for $5 \mathrm{~min}$. Expression levels were analyzed using the $2^{-\Delta \Delta C q}$ method (19) and the relative expression levels of TINCR, large tumor suppressor kinase 1 (LATS1), cellular communication network factor 2 (CTGF), cellular communication network factor 1 (CCN1), AXL receptor tyrosine kinase (AXL) and miR-424-5p were normalized to GAPDH and U6, respectively. The primer sequences are listed in Table I.

Western blotting. Total protein was extracted from A375 and MV3 cells using RIPA lysis buffer (Beyotime Institute of Biotechnology). Protein concentration was quantified using a BCA assay kit (Beyotime Institute of Biotechnology) and $20 \mu \mathrm{g}$ protein/lane was separated via $10 \%$ SDS-PAGE. The separated proteins were subsequently transferred onto PVDF membranes and blocked with 5\% non-fat milk for $2 \mathrm{~h}$ at room temperature. The membranes were then incubated with the 
Table I. Primer sequences used for reverse transcription-quantitative PCR.

\begin{tabular}{lll}
\hline Gene & \multicolumn{1}{c}{ Forward } & \multicolumn{1}{c}{ Reverse } \\
\hline miR-424-5p & 5'-GCGGCGGCAGCAGCAATTCATG-3' & 5'-ATCCAGTGCAGGGTCCGAGG-3' \\
U6 & 5'-CTCGCTTCGGCAGCACATA-3' & 5'-AACGATTCACGAATTTGCGT-3' \\
TINCR & 5'-GGACAACCTTAGCGTGTTCA-3' & 5'-TTGGATCAAAGAAGGGAAGG-3' \\
LATS1 & 5'-AAACCAGGGAATGTGCAGCAA-3' & 5'-CATGCCTCTGAGGAACTAAGGA-3' \\
CTGF & 5'-AAAAGTGCATCCGTACTCCCA-3' & 5'-CCGTCGGTACATACTCCACAG-3' \\
CCN1 & 5'-GGTCAAAGTTACCGGGCAGT-3' & 5'-GGAGGCATCGAATCCCAGC-3' \\
AXL & 5'-ATCAGCTTCGGCTAGGCAG-3' & 5'-TCCGCGTAGCACTAATGTTCT-3' \\
GAPDH & 5'-GAAGGTGAAGGTCGGAGTC-3' & 5'-GAAGATGGTGATGGGATTTC-3'
\end{tabular}

miR, microRNA; TINCR, TINCR ubiquitin domain containing; LATS1, large tumor suppressor kinase 1; CTGF, cellular communication network factor 2; CCN1, cellular communication network factor 1; AXL, AXL receptor tyrosine kinase.

following primary antibodies at $4^{\circ} \mathrm{C}$ overnight: Anti-LATS1 (1:1,000; cat. no. ab243656; Abcam), anti-phosphorylated (p)-Yes 1 associated transcriptional regulator (YAP; 1:1,000; cat. no. ab76252; Abcam), anti-YAP (1:1,000; cat. no. ab52771; Abcam) and anti- $\beta$-actin (1:1,000; cat. no. ab8226; Abcam). Following the primary antibody incubation, the membranes were incubated with HRP-conjugated anti-mouse $(1: 10,000$; cat.no.ab6289; Abcam) or anti-rabbit (1:10,000; cat. no. ab6721; Abcam) secondary antibodies for $2 \mathrm{~h}$ at room temperature. Protein bands were visualized using an ECL reagent (Pierce; Thermo Fisher Scientific, Inc.) and densitometric analysis was performed using ImageJ software (version 1.8.0; National Institutes of Health). $\beta$-actin was used as the internal loading control.

Cell Counting Kit-8 (CCK-8) assay. The proliferation of A375 and MV3 cells was measured following 1, 2, 3 and 4 days of transfection, respectively. After the transfection, $10 \mu$ l CCK-8 reagent (DojindoDojindo Molecular Technologies, Inc.) was added into each well and further incubated for $4 \mathrm{~h}$ at $37^{\circ} \mathrm{C}$, according to the manufacturer's instructions. The optical density value was measured at a wavelength of $450 \mathrm{~nm}$ using a microplate reader (Bio-Rad Laboratories, Inc.).

Flow cytometric analysis of apoptosis. A375 and MV3 cells were seeded into 12 -well plates $\left(1 \times 10^{5}\right.$ cells/well) and cultured for $48 \mathrm{~h}$ at $37^{\circ} \mathrm{C}$ prior to being harvested via digestion using $0.025 \%$ trypsin (Thermo Fisher Scientific, Inc.). The cells were washed with PBS and subsequently incubated with $5 \mu \mathrm{l}$ Annexin V-FITC and $5 \mu \mathrm{l}$ PI from an Annexin V/Dead Cell Apoptosis kit (Thermo Fisher Scientific, Inc.) for $15 \mathrm{~min}$ in the dark at $37^{\circ} \mathrm{C}$. Apoptotic cells were analyzed within $1 \mathrm{~h}$ using a FACSCalibur flow cytometer (BD Biosciences). The results were analyzed using FlowJo software version 10.2 (FlowJo LLC). The apoptotic rate was calculated as the percentage of early + late apoptotic cells. In total, 10,000 events were analyzed in each group.

Transwell assay. The invasion of A375 and MV3 cells was analyzed using Matrigel Transwell chambers (8- $\mu \mathrm{m}$ pore size; BD Biosciences). Briefly, the upper Transwell chamber was precoated with $100 \mu 1$ Matrigel (BD Biosciences) at $37^{\circ} \mathrm{C}$ for
5 h. Then, $2 \times 10^{5}$ cells suspended in serum-free RPMI-1640 medium were plated into the upper chamber. The lower chamber was filled with RPMI-1640 medium supplemented with $20 \%$ FBS. Following $48 \mathrm{~h}$ of incubation, the cells remaining in the upper chamber were removed, while the invasive cells in the lower chamber were fixed with $4 \%$ formaldehyde for $15 \mathrm{~min}$ at room temperature and stained with $0.1 \%$ crystal violet for $20 \mathrm{~min}$ at room temperature. The invasive cells were visualized (magnification, x100) using a light microscope.

Dual luciferase reporter assay. The 3'untranslated region (UTR) of LATS1 was cloned into pGL3 dual luciferase reporter vector (pGL3-LATS1; Promega Corporation). The binding sites between TINCR and miR-424-5p were predicted using miRDB (http://mirdb.org). The wild-type (WT) 3'UTR of TINCR was cloned into a pGL3 vector (pGL3-TINCR-WT). In addition, two site mutations were introduced into the pGL3-TINCR-WT sequence using a Quick Site-Directed Mutation kit (Agilent Technologies, Inc.) to generate the pGL3-TINCR-mutant (pGL3-TINCR-MUT) vector. To study the association between miR-424-5p and TINCR, cells ( $1 \times 10^{5}$ cells/well) were co-transfected with pGL3-TINCR-WT (0.4 mg) or pGL3-TINCR-MUT (0.4 mg) and miR-424-5p mimic $(20 \mathrm{mM})$ or miR-NC mimic $(20 \mathrm{mM})$ using Lipofectamine 3000 (Invitrogen; Thermo Fisher Scientific, Inc.) at $37^{\circ} \mathrm{C}$ for $48 \mathrm{~h}$. To investigate the association between TINCR, miR-424-5p and LATS1, cells were transfected with pGL3-LATS1 and miR-424-5p mimic or miR-NC mimic and pcDNA3.1-TINCR. Following $48 \mathrm{~h}$ of transfection, the relative firefly and Renilla luciferase activities were determined using a Dual Luciferase Reporter assay system (Promega Corporation). The firefly luciferase activity was normalized to Renilla luciferase activity.

Statistical analysis. Statistical analysis was performed using GraphPad Prism 6.0 software (GraphPad Software, Inc.) and the data are presented as the mean \pm SD. Paired Student's t-tests were used to determine the statistical differences between normal and tumor tissues, while unpaired Student's t-tests were used to determine statistical differences between two experimental groups of cells. A one-way ANOVA followed by a Tukey's post hoc test was used to determine the statistical 
Table II. Association between clinicopathological characteristics and TINCR expression in patients with cutaneous malignant melanoma $(n=60)$.

TINCR

\begin{tabular}{|c|c|c|c|c|}
\hline \multirow[b]{2}{*}{ Clinicopathological factor } & \multirow[b]{2}{*}{ Patients $(n=60)$} & & \multirow[b]{2}{*}{ P-value } \\
\hline & & $\operatorname{High}(\mathrm{n}=30)$ & Low $(n=30)$ & \\
\hline Age, years & & & & 0.601 \\
\hline$\leq 60$ & 35 & 16 & 19 & \\
\hline$>60$ & 25 & 14 & 11 & \\
\hline Sex & & & & 0.361 \\
\hline Male & 46 & 21 & 25 & \\
\hline Female & 14 & 9 & 5 & \\
\hline TNM stage & & & & $0.011^{\mathrm{a}}$ \\
\hline I-II & 13 & 11 & 2 & \\
\hline III-IV & 47 & 19 & 28 & \\
\hline Distant metastasis & & & & 0.779 \\
\hline Yes & 18 & 8 & 10 & \\
\hline No & 42 & 22 & 20 & \\
\hline
\end{tabular}

${ }^{\mathrm{a}} \mathrm{P}<0.05$. TINCR, TINCR ubiquitin domain containing.

differences between $\geq 3$ groups. Pearson correlation analysis was used to study the association between expression levels of miR-424-5p and TINCR in CMM samples from TCGA-SKCM using StarBase V2.0 (http://starbase.sysu.edu.cn/). This analysis was also used to study the expression levels of miR-424-5p and TINCR in the collected CMM samples. The categorical data in Table II were analyzed using a $\chi^{2}$ test. Every experiment was repeated three times. $\mathrm{P}<0.05$ was considered to indicate a statistically significant difference.

\section{Results}

TINCR expression is downregulated in CMM tissues. Analysis of RNA-seq data representing 27 different tissues from 95 human individuals revealed that the IncRNA, TINCR, was found to be predominantly expressed in skin tissue (Fig. 1A). Analysis of the expression data of TINCR in tumor and normal tissues from Gene Expression Profiling Interactive Analysis database (http://gepia.cancer.pku.cn) identified that the expression levels of TINCR were significantly downregulated in CMM tissues $(n=461)$ compared with the normal tissues $(n=558)$ (Fig. 1B). Using the GSE4587 dataset, TINCR expression was also found to be markedly downregulated in benign nevus and melanoma in situ tissues, while the lowest TINCR expression was observed in melanomas with lymph node metastasis (Fig. 1C). Similar to these results, in the tissues collected from patients with CMM, TINCR expression was downregulated in tumor tissues compared with the adjacent normal tissues ( $n=60$; Fig. 1D). Patients with CMM were divided into low $(n=30)$ and high expression $(n=30)$ groups according to the median expression value of TINCR. TINCR expression levels were not associated with age, sex or distant metastasis, while low TINCR expression was associated with an advanced TNM stage (Table II). These results suggested that TINCR may play a tumor suppressive role in the development of CMM.
TINCR overexpression reduces proliferation and invasion, and induces apoptosis in CMM cell lines. Compared with the human immortalized keratinocytes, HaCaT, TINCR expression levels were significantly downregulated in the human CMM cell lines, M14, A375 and MV3 (Fig. 2A). As A375 and MV3 cells showed relatively lower TINCR expression levels compared with M14 cells, these two cell lines were selected for use in the subsequent experiments.

In A375 and MV3 cells, compared with the control group, TINCR expression levels were upregulated following the overexpression of TINCR (Fig. 2B). In addition, the overexpression of TINCR significantly reduced cell proliferation (Fig. 2C), induced cell apoptosis (Fig. 2D) and reduced cell invasion (Fig. 2E).

miR-424-5p interacts with TINCR in CMM cell lines. Data from TCGA-SKCM dataset identified a weak negative correlation $(r=-0.247)$ between TINCR and miR-424-5p expression in CMM tissues (Fig. 3A). In the tissues collected from patients with CMM, miR-424-5p expression levels were found to be upregulated in CMM tissues compared with the normal tissues (Fig. 3B). Similarly, a moderate negative correlation ( $\mathrm{r}=-0.658)$ was also noted between TINCR and miR-424-5p expression in the CMM tissues (Fig. 3C).

The regulatory relationship between TINCR and miR-424-5p was further investigated in CMM cell lines, A375 and MV3. The expression levels of miR-424-5p were downregulated in the TINCR overexpression group compared with the control group (Fig. 3D). Next, the expression of miR-424-5p was knocked down using a miR-424-5p inhibitor, and the results revealed that the expression levels of miR-424-5p were lower in the miR-424-5p inhibitor group compared with the miR-NC inhibitor group (Fig. 3E). Transfection with the miR-424-5p inhibitor upregulated TINCR expression levels compared with the miR-NC inhibitor group (Fig. 3F). These findings suggested that TINCR and miR-424-5p may repress 
A

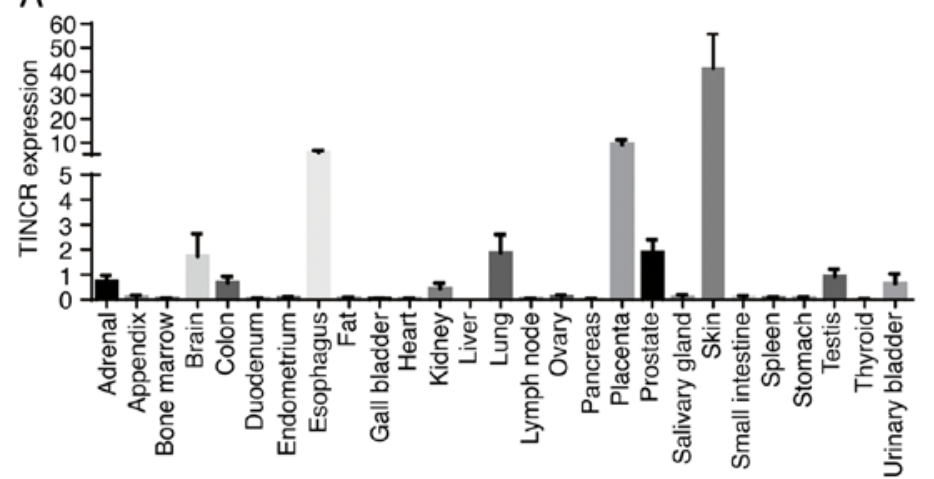

B

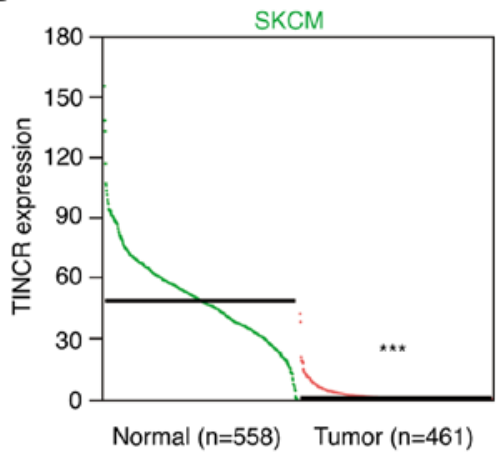

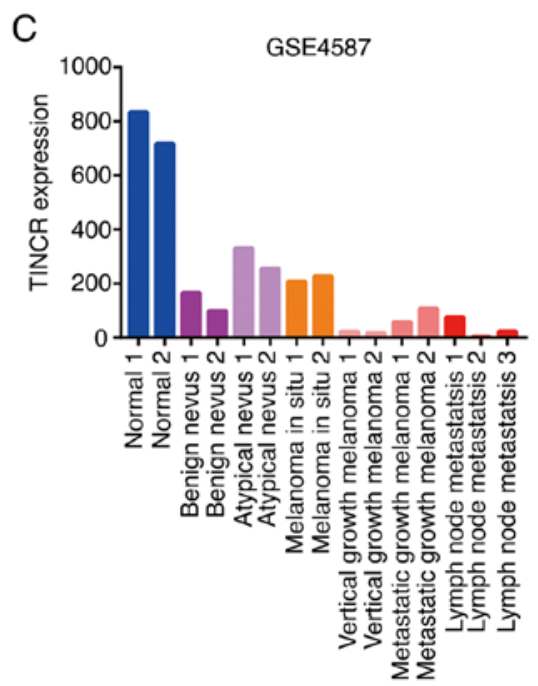

D

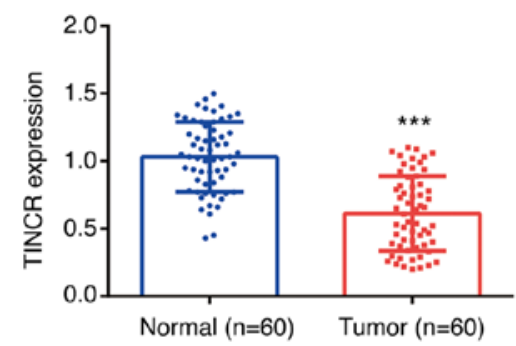

Figure 1. TINCR expression is downregulated in CMM tumor tissues. (A) TINCR expression pattern in 95 human individuals, representing 27 different tissues, was retrieved from Human Protein Atlas RNA-sequencing normal tissues project. (B) TINCR expression in CMM tumor tissues and normal tissues was retrieved from The Cancer Genome Atlas-SKCM dataset. (C) TINCR expression in normal skin, benign nevus, atypical nevus, melanoma in situ, vertical growth melanoma and lymph node metastasis melanoma was analyzed based on GSE4587 dataset. (D) TINCR expression in tumor tissues and the adjacent normal tissues was detected via reverse transcription-quantitative PCR. ${ }^{* * *} \mathrm{P}<0.001$ vs. normal tissues. TINCR, TINCR ubiquitin domain containing; CMM, cutaneous malignant melanoma; SKCM, skin cutaneous melanoma.

the expression of each other in the CMM cell lines, A375 and MV3.

The relationship between TINCR and miR-424-5p was further verified in CMM cell lines, A375 and MV3, using a dual luciferase reporter assay. miR-424-5p was successfully overexpressed using a miR-424-5p mimic, which was evidenced by the higher miR-424-5p expression in the miR-424-5p mimic group compared with the miR-NC mimic group (Fig. 3G). The binding sites between TINCR and miR-424-5p are presented in Fig. 3H. In A375 and MV3 cells transfected with the pGL3-TINCR-WT vector, the relative luciferase activity was discovered to be significantly decreased following the co-transfection with the miR-424-5p mimic compared with the miR-NC mimic (Fig. 3I). However, in A375 and MV3 cells transfected with the pGL3-TINCR-MUT vector, the relative luciferase activity was not significantly different between the miR-424-5p mimic and miR-NC mimic groups (Fig. 3J). These findings indicated that miR-424-5p may interact with TINCR in the CMM cell lines, A375 and MV3.

TINCR upregulates LATS1 expression by sponging miR-424-5p in CMM cell lines. In A375 and MV3 cells, TINCR overexpression upregulated the mRNA expression levels of LATS1 (Fig. 4A), as well as the protein expression levels of LATS1 and the p-YAP/YAP ratio, compared with the control group (Fig. 4B and C). Next, expression levels of YAP signaling-related molecules were detected. In A375 and MV3 cells, TINCR overexpression downregulated the mRNA expression levels of CTGF, CCN1 and AXL compared with the control group (Fig. 4D and E). These results suggested the potential positive regulatory relationships between TINCR and LATS1, TINCR and the p-YAP/YAP ratio, as well as TINCR and YAP signaling-related molecules.

In A375 and MV3 cells, compared with the miR-NC mimic group, transfection with the miR-424-5p mimic significantly downregulated the mRNA and protein expression levels of LATS1, which were then rescued by TINCR overexpression (Fig. 4F and G). Similar trends were also observed in the relative luciferase activity of the LATS1 3'UTR vector (Fig. 4H). These findings suggested the existence of a regulatory relationship among TINCR, miR-424-5p and LATS1.

TINCR reduces the proliferation and invasion, and induces the apoptosis of CMM cell lines by regulating LATS1 expression. In A375 and MV3 cells, compared with the siControl group, siLATS1 downregulated the mRNA expression levels of 

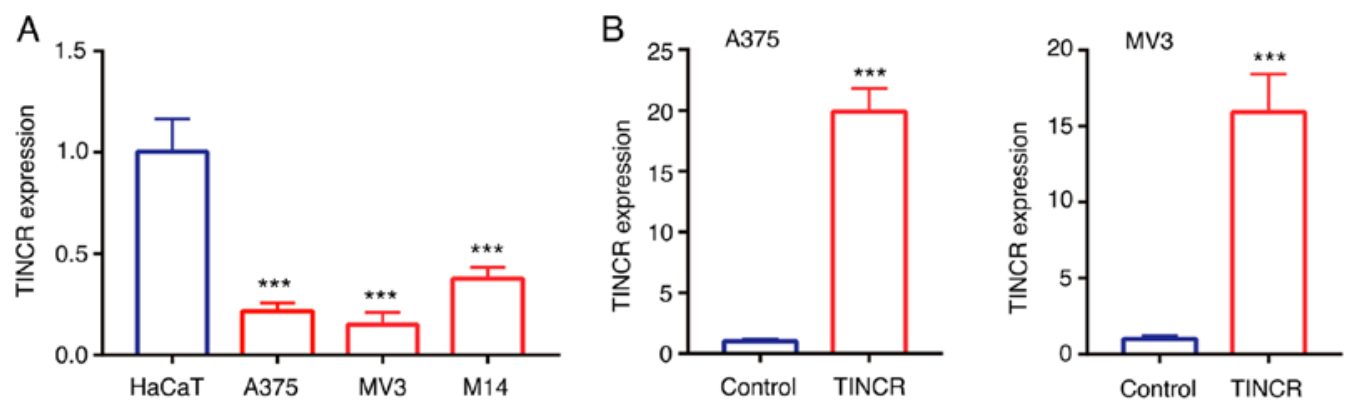

C
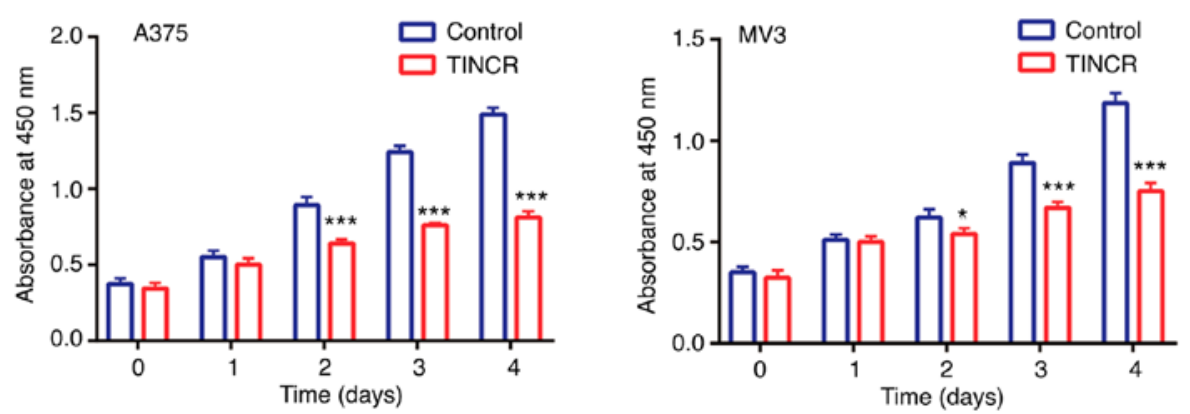

D
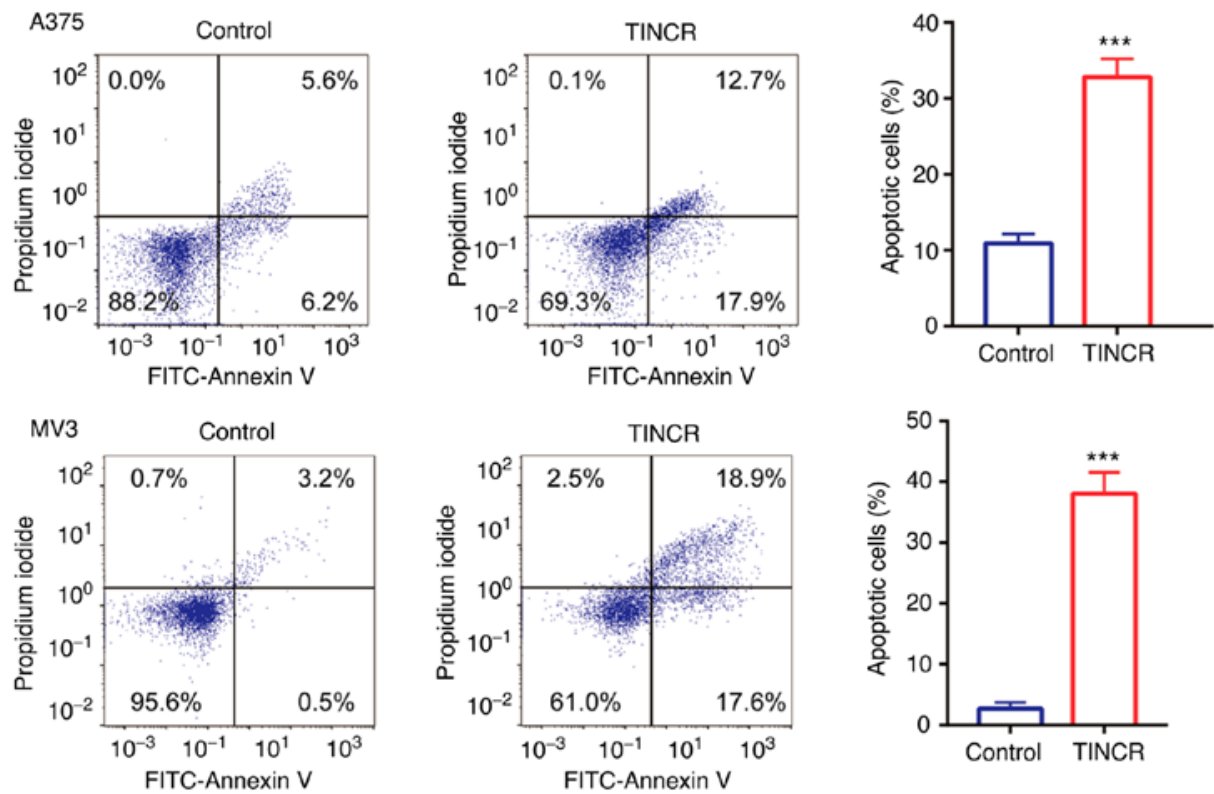

E
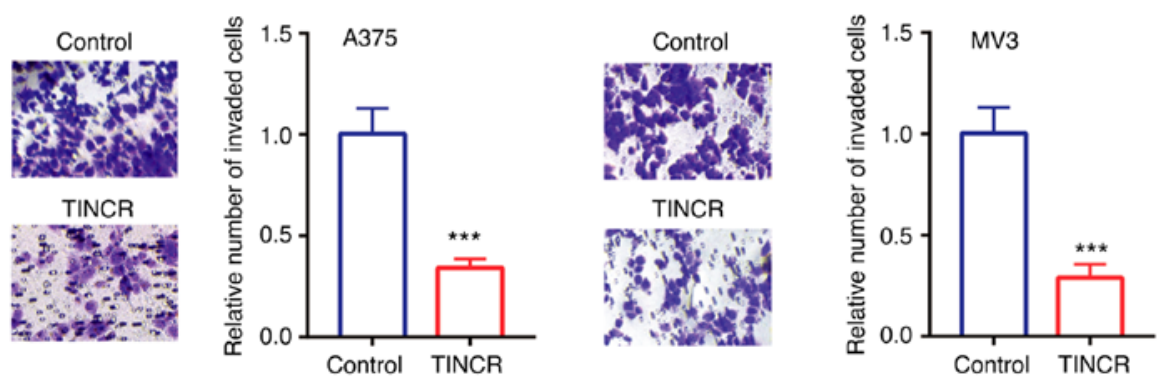

Figure 2. TINCR overexpression reduces proliferation and induces apoptosis in CMM cell lines. (A) TINCR expression in HaCaT cells and CMM cell lines, including M14, A375 and MV3, was detected via RT-qPCR. ${ }^{* * *} \mathrm{P}<0.001$ vs. HaCaT cells. (B) In A375 and MV3 cells, TINCR expression in control group and TINCR overexpression group was examined via RT-qPCR. (C) Cell proliferation in control group and TINCR overexpression group was examined using Cell Counting Kit-8 assays. (D) Cell apoptosis in control group and TINCR overexpression group was examined via flow cytometry. (E) Cell invasion in control group and TINCR overexpression group was examined via Transwell assays (magnification, $\mathrm{x} 100)$. ${ }^{*} \mathrm{P}<0.05,{ }^{* * * *} \mathrm{P}<0.001 \mathrm{vs}$. control (pcDNA3.1). RT-qPCR, reverse transcription-quantitative PCR; TINCR, TINCR ubiquitin domain containing; CMM, cutaneous malignant melanoma.

LATS1 (Fig. 5A). Moreover, in A375 and MV3 cells, compared with the control group, TINCR overexpression upregulated the mRNA expression levels of LATS1, which were reversed following the co-transfection with siLATS1 (Fig. 5B). These findings further indicated the positive relationship between TINCR and LATS1 in CMM cell lines, A375 and MV3. 


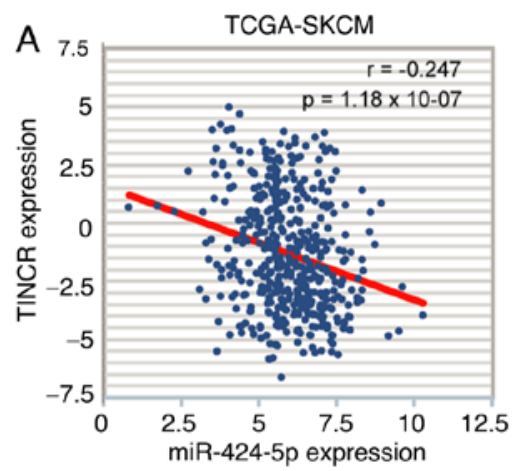

B

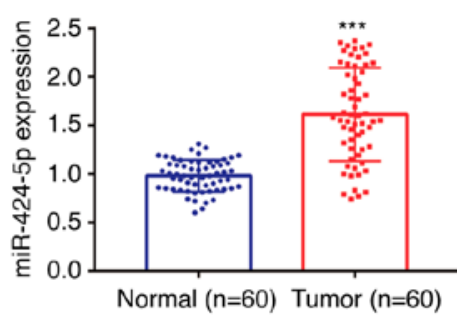

E

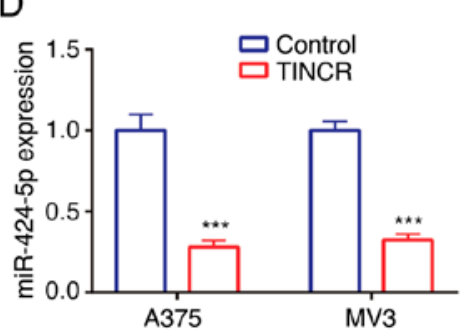

G

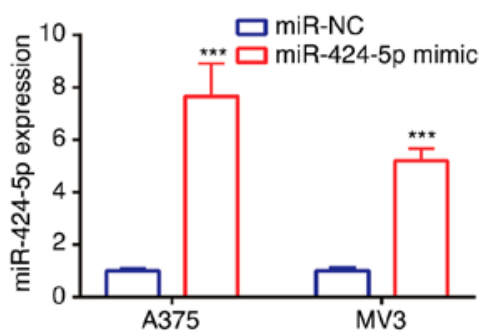

I

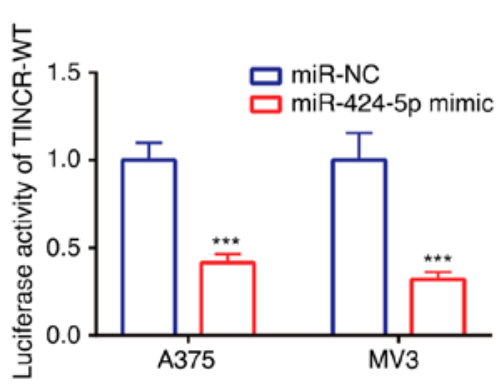

$\mathrm{H}$
C

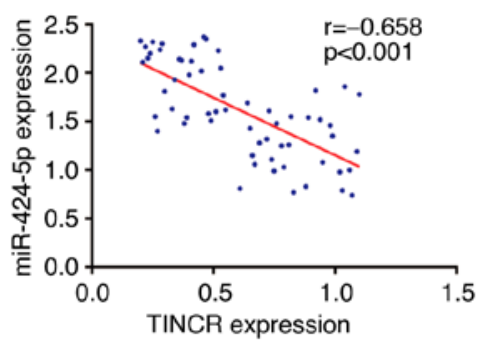

F

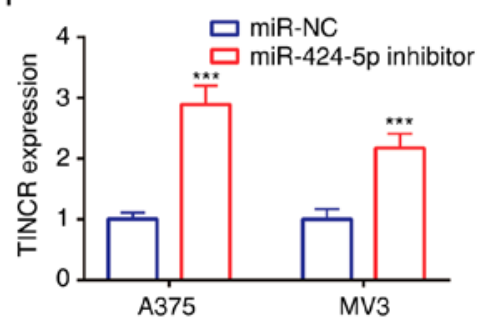

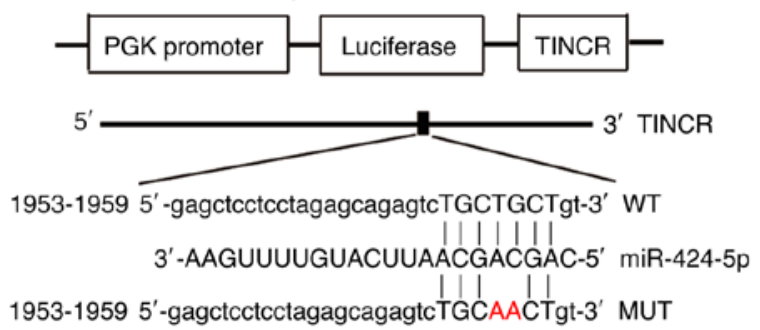

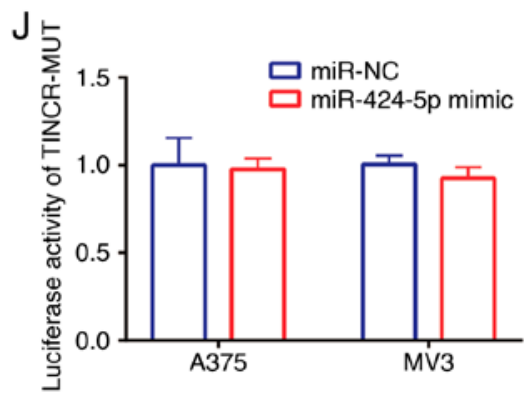

Figure 3. miR-424-5p interacts with the long non-coding RNA TINCR in CMM cell lines. (A) Correlation between TINCR and miR-424-5p in CMM tumor tissues retrieved from The Cancer Genome Atlas-SKCM project. (B) miR-424-5p expression in tumor tissues and the adjacent normal tissues was detected via RT-qPCR. ${ }^{* * *} \mathrm{P}<0.001$ vs. normal. (C) Correlation between TINCR and miR-424-5p expression in CMM tumor tissues was analyzed via Pearson correlation analysis. (D) miR-424-5p expression in control group and TINCR overexpression group was detected via RT-qPCR. ${ }^{* * *} \mathrm{P}<0.001$ vs. control. (E) miR-424-5p and (F) TINCR expression in miR-NC group and miR-424-5p inhibitor groups was detected via RT-qPCR. (G) miR-424-5p expression in miR-NC group and miR-424-5p mimic group was detected via RT-qPCR. (H) Binding sites between TINCR and miR-424-5p were predicted using miRDB (H). Luciferase activity in A375 and MV3 cells that were transfected with (I) TINCR-WT and (J) TINCR-MUT in miR-424-5p mimic group and miR-NC group was detected using a dual luciferase activity assay. ${ }^{* * *} \mathrm{P}<0.001$ vs. miR-NC. TINCR, TINCR ubiquitin domain containing; CMM, cutaneous malignant melanoma; SKCM, skin cutaneous melanoma; WT, wild-type; MUT, mutant; miR, microRNA; NC, negative control; RT-qPCR, reverse transcription-quantitative PCR.

The functions of TINCR and LATS1 in CMM cell lines were subsequently investigated. In A375 cells, compared with the control group, TINCR overexpression significantly reduced proliferation (Fig. 5C), induced apoptosis (Fig. 5D) and decreased invasion (Fig. 5E), which were all partially reversed following the co-transfection with siLATS1. Similar effects were observed in the proliferation (Fig. 5F), apoptosis (Fig. 5G) and invasion (Fig. 5H) of MV3 cells following combined TINCR overexpression and siLATS1 transfection.

\section{Discussion}

The metastasis of melanoma further promotes the progression of the disease (20). The results of the present study revealed that TINCR expression levels were downregulated in CMM tissues from the GSE4587 dataset obtained from the GEO database, as well as in collected CMM tissues. Low expression of TINCR was observed in tumors of advanced stage in comparison with those of early stage. TINCR overexpression was found to decrease the proliferation and invasion, and 

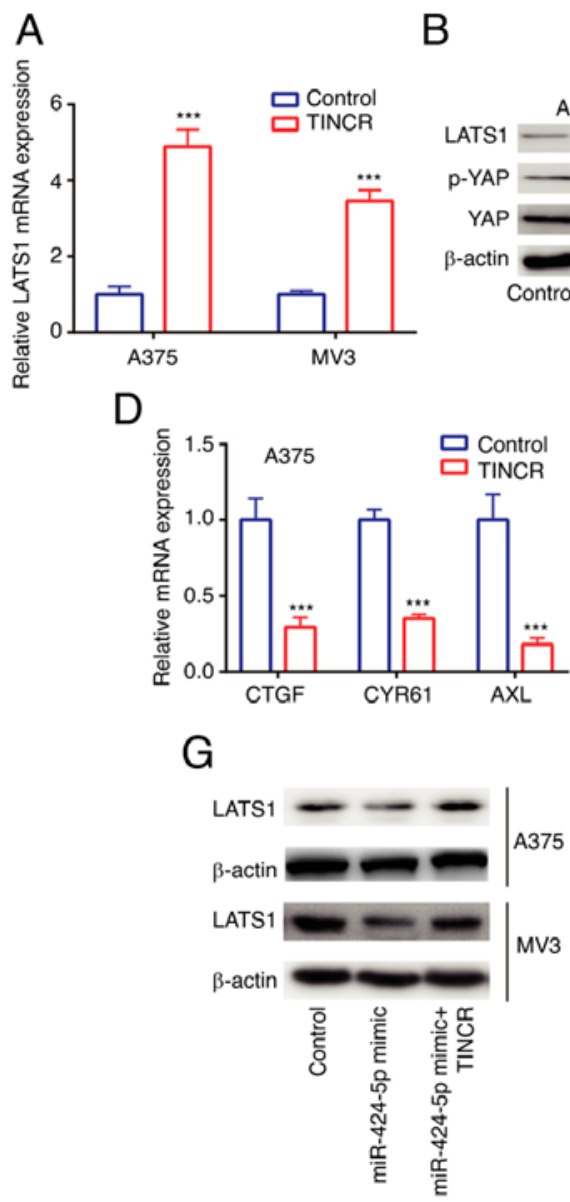

B
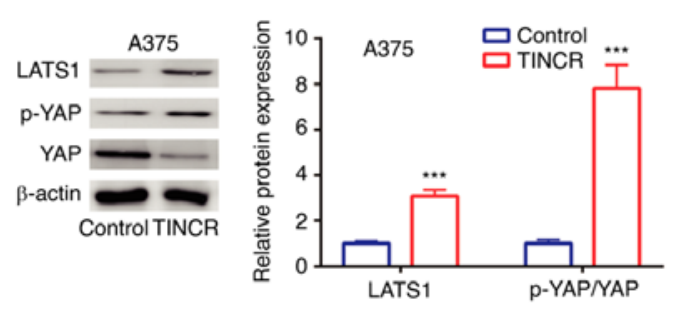

E
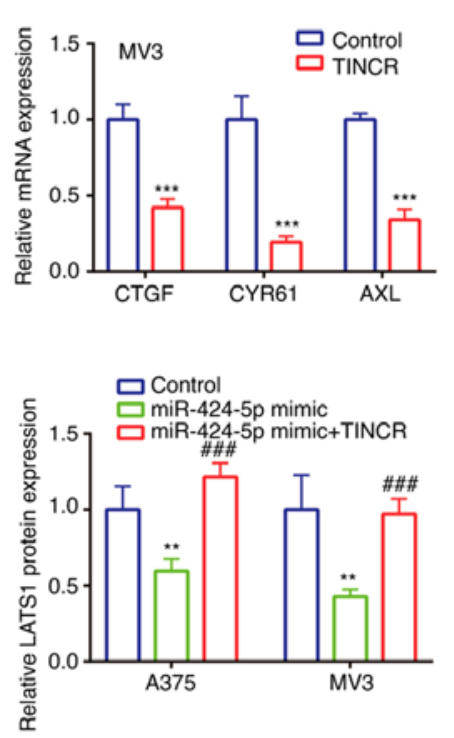

C
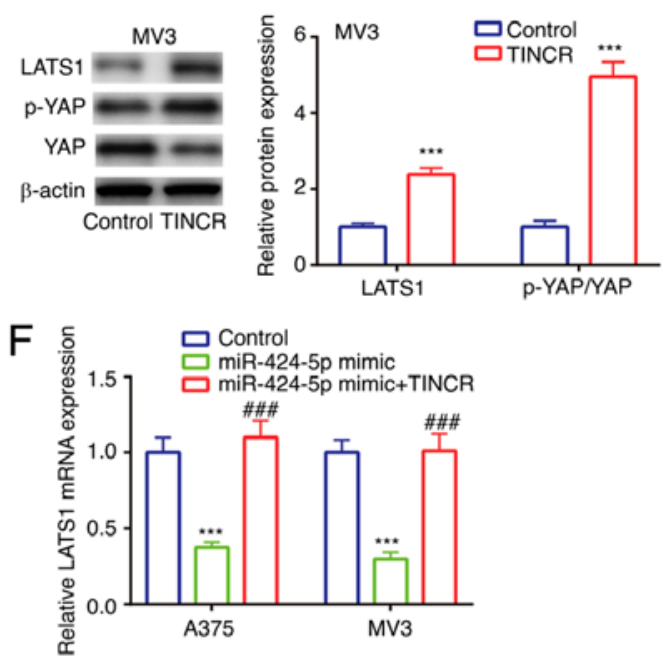

$\mathrm{H}$

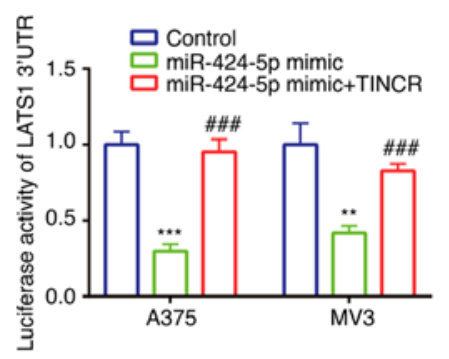

Figure 4. TINCR promotes LATS1 expression by sponging miR-424-5p in cutaneous malignant melanoma cell lines. (A) LATS1 mRNA expression in control group and TINCR overexpression group was determined via RT-qPCR. LATS1 protein expression and p-YAP/YAP ratio in control group and TINCR overexpression group were determined via western blotting in (B) A375 and (C) MV3 cells. CTGF, CCN1 and AXL mRNA expression in control group and TINCR overexpression group was determined via RT-qPCR in (D) A375 and (E) MV3 cells. LATS1 (F) mRNA and (G) protein expression in control group, miR-424-5p mimic group and miR-424-5p mimic + TINCR group was determined via RT-qPCR and western blotting. (H) Luciferase activity of LATS1 3'UTR in control group, miR-424-5p mimic group and miR-424-5p mimic + TINCR group was determined using a dual luciferase activity assay. ${ }^{* *} \mathrm{P}<0.01,{ }^{* * *} \mathrm{P}<0.001$ vs. control (pcDNA3.1, miR-NC mimic + pcDNA3.1); ${ }^{\# \# ~ P<0.001 ~ v s . ~ m i R-424-5 p ~ m i m i c . ~ m i R, ~ m i c r o R N A ; ~ R T-q P C R, ~ r e v e r s e ~ t r a n s c r i p t i o n-q u a n t i t a t i v e ~ P C R ; ~ T I N C R, ~ T I N C R ~}$ ubiquitin domain containing; LATS1, large tumor suppressor kinase 1; p-, phosphorylated; YAP, Yes 1 associated transcriptional regulator; UTR, untranslated region; CTGF, cellular communication network factor 2; CCN1, cellular communication network factor 1; AXL, AXL receptor tyrosine kinase.

induce the apoptosis of CMM cell lines, indicating the potential tumor suppressive role of TINCR in CMM.

It is well-known that lncRNAs regulate gene expression by promoting RNA degradation, miRNA sequestration and transcriptional and translational activation or repression (10). Using next generation sequencing, a previous study discovered that TINCR was negatively correlated with miR-424-5p expression in CMM tissues, which was found to be associated with the invasive and aggressive phenotype of CMM (21). However, to the best of our knowledge, studies reporting the precise function of miR-424-5p or the correlation between TINCR and miR-424-5p in the development of CMM have not been conducted.

miR-424-5p has been shown to function as an oncogene in numerous cancer types. For example, miR-424-5p promoted lung metastasis in thyroid cancer via inactivation of the Hippo signaling pathway (22); miR-424-5p increased cell proliferation in gastric cancer by targeting $\mathrm{Smad} 3$ via regulation of TGF- $\beta$ signaling (23); and serum miR-424-5p expression levels were found to be increased in patients with colorectal cancer (24). Consistent with the reported oncogenic role of
miR-424-5p in the aforementioned cancer types, miR-424-5p expression levels were also discovered to be upregulated in CMM tissues in the current study. In addition, miR-424-5p was identified to interact with TINCR in CMM cell lines, indicating the oncogenic role of miR-424-5p in the progression of CMM. Based on these results, the expression of target genes of miR-424-5p that were regulated by TINCR were analyzed.

LATS1 was previously identified as a target of miR-424-5p (25) and was reported to serve a tumor suppressive role in numerous cancer types; for example, LATS1 suppressed the development of breast cancer by maintaining cell identity (26); miR-103a-3p induced the malignant progression of thyroid cancer via Hippo signaling by targeting LATS1 (27); and previous mutation analyses on LATS1 highlighted its tumor suppressive role in numerous human cancer types, including stomach adenocarcinoma, uterine corpus endometrial carcinoma and bladder urothelial carcinoma (28). The results of the present study revealed that LATS1 expression was positively regulated by TINCR in CMM cell lines. Subsequently, the functional relationship between TINCR and LATS1 in CMM cell lines was investigated. 
A

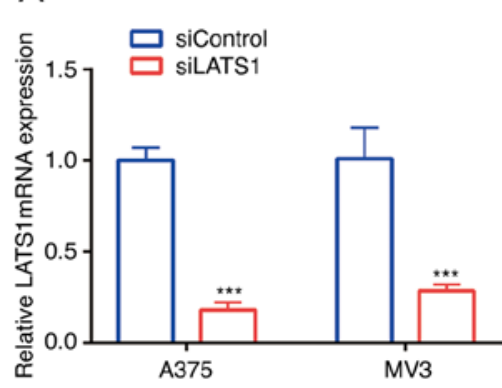

B 口 Control

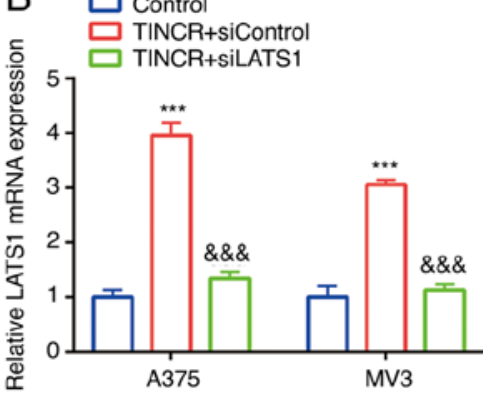

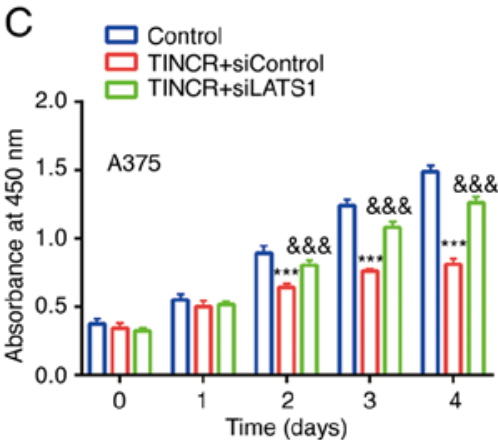
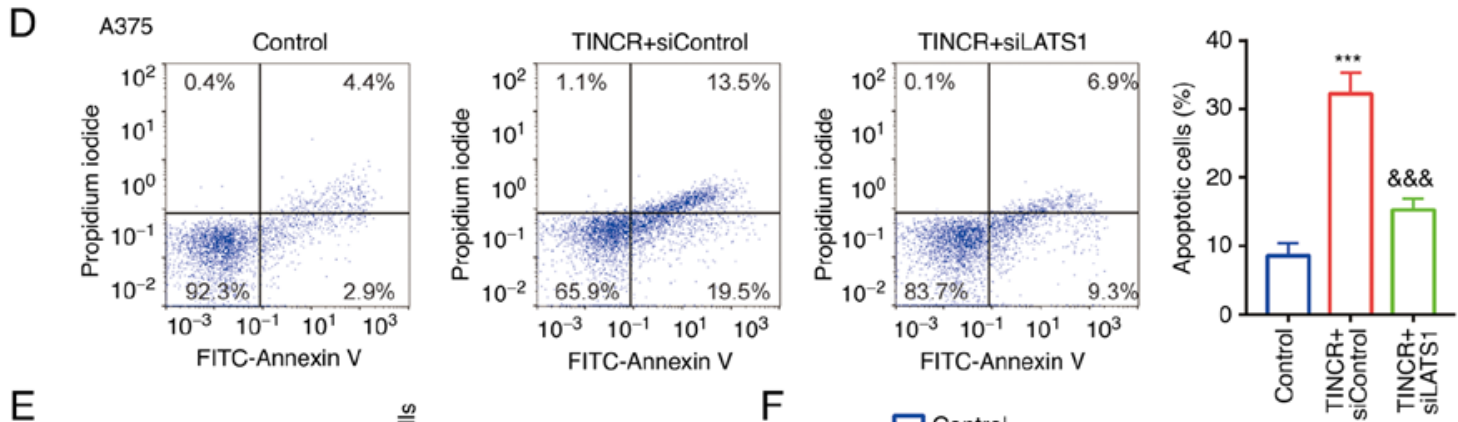

E
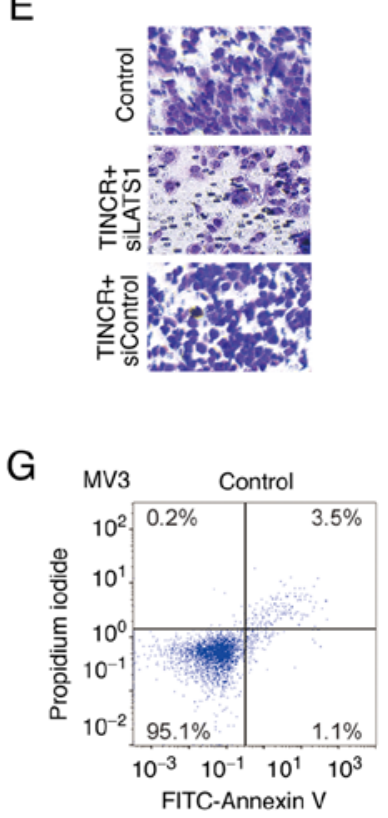

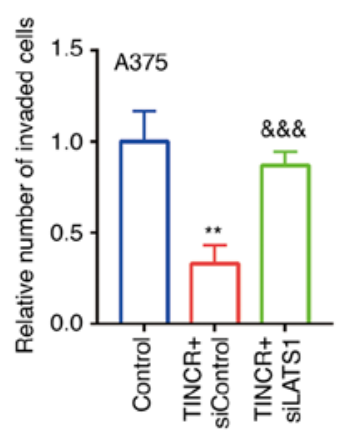

$\mathrm{F}$

\section{只Control}

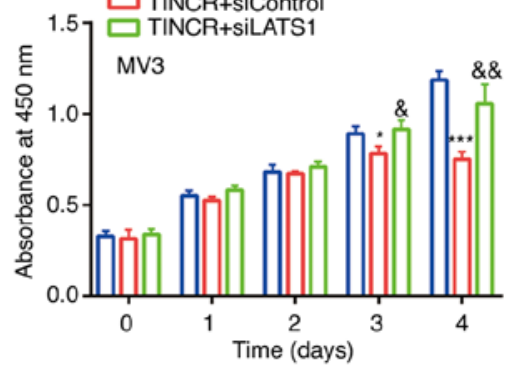

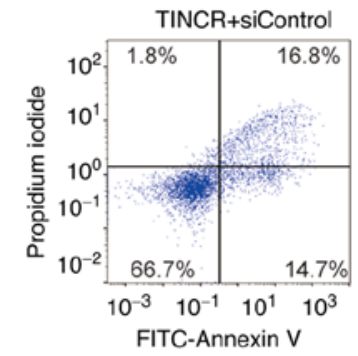
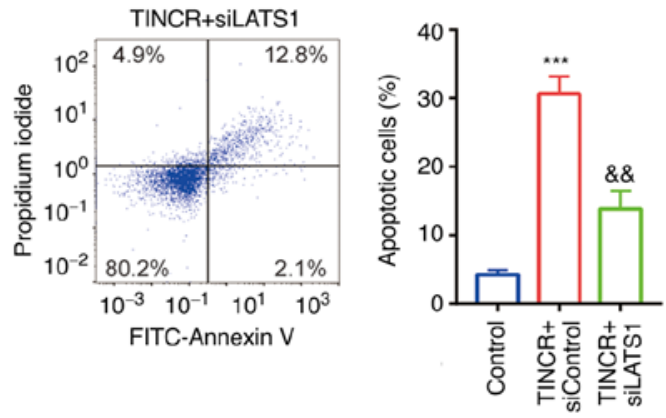
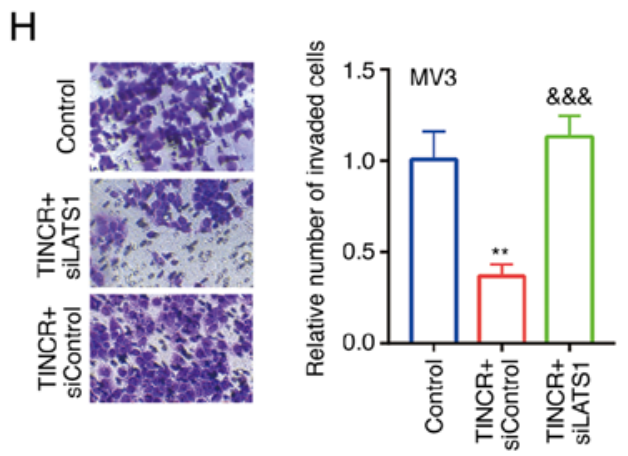

Figure 5. TINCR reduces the proliferation and induces the apoptosis of CMM cell lines by regulating LATS1. (A) LATS1 mRNA expression in siControl group and siLATS1 group was determined via RT-qPCR. ${ }^{* * * *} \mathrm{P}<0.001$ vs. siControl. (B) LATS1 mRNA expression in control group, TINCR + siControl group and TINCR + siLATS1 group was determined via RT-qPCR. (C) In A375 cells, proliferation in control group, TINCR + siControl group and TINCR+ siLATS1 group was examined using a Cell Counting Kit-8 assay. (D) Cell apoptosis in control group, TINCR + siControl group and TINCR+ siLATS1 group was examined via flow cytometry. (E) Cell invasion in control group, TINCR + siControl group and TINCR + siLATS1 group was examined using Transwell assays (magnification, x100). (F) In MV3 cells, proliferation in control group, TINCR + siControl group and TINCR + siLATS1 group was examined using a Cell Counting Kit-8 assay. (G) Cell apoptosis in control group, TINCR + siControl group and TINCR + siLATS1 group was examined via flow cytometry. (H) Cell invasion in control group, TINCR + siControl group and TINCR + siLATS1 group was examined using a Transwell assay (magnification, $\mathrm{x} 100)$. " $\mathrm{P}<0.05$, ${ }^{* *} \mathrm{P}<0.01,{ }^{* * *} \mathrm{P}<0.001$ vs. control (pcDNA3.1 + siControl); ${ }^{\circledR} \mathrm{P}<0.05$, ${ }^{\&}{ }^{\&} \mathrm{P}<0.01$, ${ }^{\text {\& \& }} \mathrm{P}<0.001$ vs. TINCR + siControl. RT-qPCR, reverse transcription-quantitative PCR; TINCR, TINCR ubiquitin domain containing; LATS1, large tumor suppressor kinase 1; si, small interfering RNA. 
LATS1 is a core member of the Hippo/YAP signaling pathway (29). The Hippo/YAP signaling pathway is known to regulate cell proliferation and invasion (30-32). AXL, CTGF and CCN1 are all downstream molecules that are associated with the Hippo/YAP signaling pathway (33). The findings of the current study demonstrated that TINCR overexpression activated Hippo signaling and repressed the activity of YAP, as well as the expression levels of AXL, CTGF and CCN1 by regulating LATS1 expression in CMM cells. Rescue assays were performed and the results revealed that LATS1 knockdown could reverse the effect of TINCR overexpression on the proliferation, invasion and apoptosis of CMM cells.

However, there remains a limitation to the present study. For instance, there were differences between cell lines in protein expression levels reported in western blot analyses, which could be attributed possibly to morphology, gene expression or other cell line characteristics. This will be addressed in future work in more detail.

In conclusion, the present data suggested that TINCR may attenuate the proliferation and invasion, and enhance the apoptosis of CMM cells by regulating the miR-424-5p/LATS1 signaling axis. These results suggested that TINCR may play a tumor suppressive role in CMM.

\section{Acknowledgements}

Not applicable.

\section{Funding}

No funding was received.

\section{Availability of data and materials}

The datasets used and/or analyzed during the current study are available from the corresponding author on reasonable request.

\section{Authors' contributions}

$\mathrm{XH}, \mathrm{YJ}, \mathrm{XC}$ and $\mathrm{CS}$ performed the experimentations and data analysis. XH, YJ and JS designed the experiments. $\mathrm{XH}$ and JS were responsible for confirming the authenticity of the raw data. JS supervised the experimentations and prepared the manuscript. All authors read and approved the final manuscript.

\section{Ethics approval and consent to participate}

All patients provided written informed consent prior to participation in the study. The current study protocol was approved by the Ethical Committee of The China-Japan Union Hospital of Ji Lin University (approval no. CJUHJLU20161102).

\section{Patient consent for publication}

Not applicable.

\section{Competing interests}

The authors declare that they have no competing interests.

\section{References}

1. Miller AJ and Mihm MC Jr: Melanoma. N Engl J Med 355: 51-65, 2006.

2. Wu Y, Wang Y, Wang L, Yin P, Lin Y and Zhou M: Burden of melanoma in China, 1990-2017: Findings from the 2017 global burden of disease study. Int J Cancer 147: 692-701, 2020.

3. Aubuchon MM, Bolt LJ, Janssen-Heijnen ML, VerleisdonkBolhaar ST, van Marion A and van Berlo CL: Epidemiology, management and survival outcomes of primary cutaneous melanoma: A ten-year overview. Acta Chir Belg 117: 29-35, 2017.

4. Karlsson P, Boeryd B, Sander S, Westermark P and Rosdahl I: Increasing incidence of cutaneous malignant melanoma in children and adolescents 12-19 years of age in Sweden 1973-92. Acta Derm Venereol 78: 289-292, 2008.

5. Veierød MB, Weiderpass E, Thörn M, Hansson J, Lund E, Armstrong B and Adami HO: A prospective study of pigmentation, sun exposure, and risk of cutaneous malignant melanoma in women. J Natl Cancer Inst 95: 1530-1538, 2003.

6. Lees VC and Briggs JC: Effect of initial biopsy procedure on prognosis in Stage 1 invasive cutaneous malignant melanoma: Review of 1086 patients. Br J Surg 78: 1108-1110, 2010.

7. Tseng WW, Fadaki N and Leong SP: Metastatic tumor dormancy in cutaneous melanoma: Does surgery induce escape? Cancers (Basel) 3: 730-746, 2011.

8. Kim JH, Hahn EW and Tokita N: Combination hyperthermia and radiation therapy for cutaneous malignant melanoma. Cancer 41: 2143-2148, 1978

9. Li PF, Chen SC, Xia T, Jiang XM, Shao YF, Xiao BX and Guo JM: Non-coding RNAs and gastric cancer. World J Gastroenterol 20: 5411-5419, 2014.

10. Moran VA, Perera RJ and Khalil AM: Emerging functional and mechanistic paradigms of mammalian long non-coding RNAs. Nucleic Acids Res 40: 6391-6400, 2012.

11. Chen X, Gao J, Yu Y, Zhao Z and Pan Y: LncRNA FOXD3-AS1 promotes proliferation, invasion and migration of cutaneous malignant melanoma via regulating miR-325/MAP3K2. Biomed Pharmacother 120: 109438, 2019.

12. Chen XE, Chen P, Chen S, Lu J, Ma T, Shi G and Sheng L: Long non-coding RNA FENDRR inhibits migration and invasion of cutaneous malignant melanoma cells. Biosci Rep 40: BSR20191194, 2020.

13. Yu S, Wang D, Shao Y, Zhang T, Xie H, Jiang X, Deng Q, Jiao Y, Yang J, Cai C and Sun L: SP1-induced lncRNA TINCR overexpression contributes to colorectal cancer progression by sponging miR-7-5p. Aging 11: 1389-1403, 2019.

14. Dong H, Hu J, Zou K, Ye M, Chen Y, Wu C, Chen X and Han M: Activation of LncRNA TINCR by H3K27 acetylation promotes Trastuzumab resistance and epithelial-mesenchymal transition by targeting MicroRNA-125b in breast cancer. Mol Cancer 18: $3,2019$.

15. Dong L, Ding H, Li Y, Xue D and Liu Y: LncRNA TINCR is associated with clinical progression and serves as tumor suppressive role in prostate cancer. Cancer Manag Res 10: 2799-2807, 2018.

16. Smith AP, Hoek K and Becker D: Whole-genome expression profiling of the melanoma progression pathway reveals marked molecular differences between nevi/melanoma in situ and advanced-stage melanomas. Cancer Biol Ther 9: 1018-29, 2005.

17. Fagerberg L, Hallström BM, Oksvold P, Kampf C, Djureinovic D, Odeberg J, Habuka M, Tahmasebpoor S, Danielsson A, Edlund $\mathrm{K}$, et al: Analysis of the human tissue-specific expression by genome-wide integration of transcriptomics and antibody-based proteomics. Mol Cell Proteomics 13: 397-406, 2014.

18. Tang Z, Li C, Kang B, Gao G, Li C and Zhang Z: GEPIA: A web server for cancer and normal gene expression profiling and interactive analyses. Nucleic Acids Res 45: W98-W102, 2017.

19. Livak KJ and Schmittgen TD: Analysis of relative gene expression data using real-time quantitative PcR and the 2(-delta deltac(T)) method. Methods 25: 402-408, 2001.

20. Mo J, Zhao X, Dong X, Liu T, Zhao N, Zhang D, Wang W, Zhang Y and Sun B: Effect of EphA2 knockdown on melanoma metastasis depends on intrinsic ephrinA1 level. Cell Oncol (Dordr) 43: 655-667, 2020.

21. Babapoor S, Wu R, Kozubek J, Auidi D, Grant-Kels JM and Dadras SS: Identification of microRNAs associated with invasive and aggressive phenotype in cutaneous melanoma by next-generation sequencing. Lab Invest 97: 636-648, 2017. 
22. Liu X, Fu Y, Zhang G, Zhang D, Liang N, Li F, Li C, Sui C, Jiang J, Lu H, et al: miR-424-5p promotes anoikis resistance and lung metastasis by inactivating Hippo signaling in thyroid cancer. Mol Ther Oncolytics 15: 248-260, 2019.

23. Wei S,Li Q,Li Z, Wang L,Zhang Land Xu Z: miR-424-5p promotes proliferation of gastric cancer by targeting Smad3 through TGF- $\beta$ signaling pathway. Oncotarget 7: 75185-75196, 2016.

24. Sahami-Fard MH, Kheirandish S and Sheikhha MH: Expression levels of miR-143-3p and -424-5p in colorectal cancer and their clinical significance. Cancer Biomark 24: 291-297, 2019.

25. Zhang J, Liu H, Hou L, Wang G, Zhang R, Huang Y, Chen X and Zhu J: Circular RNA_LARP4 inhibits cell proliferation and invasion of gastric cancer by sponging miR-424-5p and regulating LATS1 expression. Mol Cancer 16: 151, 2017.

26. Furth N, Pateras IS, Rotkopf R, Vlachou V, Rivkin I, Schmitt I Bakaev D, Gershoni A, Ainbinder E, Leshkowitz D, et al: LATS1 and LATS2 suppress breast cancer progression by maintaining cell identity and metabolic state. Life Sci Alliance 1: e201800171, 2018

27. Zhang ML, Sun WH, Wu HQ, Liu ZD and Wang P: Knockdown of microRNA-103a-3p inhibits the malignancy of thyroid cancer cells through Hippo signaling pathway by upregulating LATS1. Neoplasma 67: 1266-1278, 2020.

28. Yu T, Bachman J and Lai ZC: Mutation analysis of large tumor suppressor genes LATS1 and LATS2 supports a tumor suppressor role in human cancer. Protein Cell 6: 6-11, 2015.
29. Zhang J, Wang G, Chu SJ, Zhu JS, Zhang R, Lu WW, Xia LQ, Lu YM, Da W and Sun Q: Loss of large tumor suppressor 1 promotes growth and metastasis of gastric cancer cells through upregulation of the YAP signaling. Oncotarget 7: 16180-16193, 2016.

30. Ehmer U and Sage J: Control of proliferation and cancer growth by the hippo signaling pathway. Mol Cancer Res 14: 127-140, 2016.

31. Janse van Rensburg HJ and Yang X: The roles of the hippo pathway in cancer metastasis. Cell Signal 28: 1761-1772, 2016.

32. Yu S, Zhang M, Huang L, Ma Z, Gong X, Liu W, Zhang J, Chen L, Yu Z, Zhao W and Liu Y: ERK1 indicates good prognosis and inhibits breast cancer progression by suppressing YAP1 signaling. Aging 11: 12295-12314, 2019.

33. Brodowska K, Al-Moujahed A, Marmalidou A, Horste M, Cichy J, Miller J, Evangelos E and Vavvas D: The clinically used photosensitizer Verteporfin (VP) inhibits YAP-TEAD and human retinoblastoma cell growth in vitro without light activation. Exp Eye Res 124: 67-73, 2014.

This work is licensed under a Creative Commons Attribution-NonCommercial-NoDerivatives 4.0 International (CC BY-NC-ND 4.0) License. 